\title{
Review: current evidence does not show a strong causal relation between the use of cannabis in young people and psychosocial harm
}

Macleod J, Oakes R, Copello A, et al. Psychological and social sequelae of cannabis and other illicit drug use by young people: a systematic review of longitudinal, general population studies. Lancet 2004;363:1579-88.

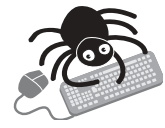

This article contains extra text on the EBMH website

Does the use of cannabis in young people have consequences for their psychological or social health?

\section{METHODS}

$\square$

Design: Systematic review.

$\square$

Data sources: MEDLINE, EMBASE, CINAHL, PsycLIT, Web of

Science, Addiction Abstracts, plus specialist databases of the

Lindesmith Center, DrugScope, US National Institute on Drug Abuse and Substance abuse, and Mental Health Services Administration, searched up to June 2003. Experts were contacted and authors of selected papers were asked for unpublished data. Non-English publications were translated.

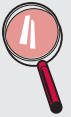

Study selection and analysis: Prospective studies measuring psychological or social harms of illicit drug use in people aged 25 years or under in the general population were eligible. Studies were categorised by quality (see notes); higher quality evidence was summarised by odds ratio (crude and adjusted for confounding factors) and descriptively. Lower quality studies were summarised and critiqued.

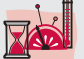

Outcomes: Educational attainment, use of other drugs, psychological health, antisocial behaviour, and other socia problems (see table for individual outcome descriptions, at http://www.ebmentalhealth.com/supplemental).

\section{MAIN RESULTS}

Sixteen out of 48 longitudinal studies were classified as of higher methodological quality. Cannabis use was consistently associated with reduced educational attainment and use of other drugs. However, when estimates were adjusted for potential confounding factors, these estimates were often substantially attenuated. Cannabis use was inconsistently associated with psychological problems and antisocial or problematic behaviour; however, adjustment of these estimates generally led to their attenuation, often substantially so. See table for the crude and adjusted effect estimates for individual studies (http:// www.ebmentalhealth.com/supplemental).

\section{CONCLUSIONS}

Using existing evidence, no causal relation can be shown between cannabis use by young people and psychosocial harm. However, this does not exclude the possibility that such a relation exists.

For correspondence: John Macleod, Department of Primary Care and Genera Practice, Primary Care Clinical Sciences and Learning Centre Building, University of Birmingham, Edgbaston, Birmingham, UK; i.a.macleod@bham. ac.uk

Sources of funding: the UK Department of Health, Drug Misuse Research Initiative.

\section{NOTES}

Studies were categorised as higher, uncertain, and lower quality. Evidence was categorised as higher quality if there was a low probability of selection bias, exposure to drugs was assessed by a validated method, and there was a follow to up period of several years and adjustments for confounding. The validity and relevance of the outcome measure was also considered.

\section{Commentary}

7 his review includes general population longitudinal studies reporting associations between cannabis use and psychosocial harm. Other substances are mentioned briefly. The search strategy is more exhaustive than in previous reviews, and relevant methodological issues are appropriately discussed. Cannabis use is associated with negative outcome on educational attainment, use of other drugs, and to some extent psychological problems. However, the authors conclude that these associations are not based on causal mechanisms. Instead it is proposed that methodological limitations prevent firm conclusions.

Different negative outcomes have been proposed to follow from cannabis use in a causal way (for example, "amotivational syndrome" and "cannabis psychosis") but there has been little empirical support for any of these hypotheses. There is general agreement that it is difficult to disentangle any specific effects of cannabis on various psychosocial outcomes, and that most of the existing studies suffer from methodological limitations. Therefore, the conclusions of the review are in accordance with other previous reviews. ${ }^{1-3} \mathrm{~A}$ notable exception is a recent review by Arseneault ${ }^{4}$ on the relation between cannabis use and schizophrenia. Here the authors conclude that cannabis use increases the risk for development of schizophrenia in a causal way. However, both reviews agree that causal effects of cannabis use for psychosocial outcomes must be limited, and the divergent conclusions rest partly on different conceptualisations of causality.

The authors suggest that the results have implications for policy practise. They argue that as cannabis cannot be causally linked with negative psychosocial outcome, harm reduction policy based on prevention of cannabis and other drug use is unlikely to produce improvements in public health. A more conservative standpoint would be to await results from further large scale studies with a sound methodology.

Mikkel Arendt, MScPsych

Centre for Basic Psychiatric Research, Aarhus University Hospital, Risskov, Aarhus, Denmark

1 Hall W, Solowij N. Adverse effects of cannabis. Lancet 1998;352:1611-16.

2 Lynskey M, Hall W. The effects of adolescent cannabis use on educational attainment: a review. Addiction 2000:95:1621-30.

3 Johns A. Psychiatric effects of cannabis. Br J Psychiatry 2001;178:116-22.

4 Arseneault L, Cannon M, Witton J, et al. Causal association between cannabis and psychosis: examination of the evidence. Br J Psychiatry 2004:184:110-17. 